\title{
Depression im Alter - was trägt die aktuelle epidemiologische Forschung bei?
}

\author{
Depression in Late Life - What does Epidemiology Add?
}

Autoren

Institut
Steffi G. Riedel-Heller, Melanie Luppa

Institut für Sozialmedizin, Arbeitsmedizin und Public Health (ISAP), Medizinische Fakultät, Universität Leipzig

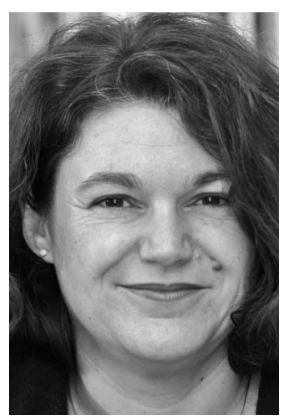

Prof. Dr. med. Steffi G. Riedel-Heller

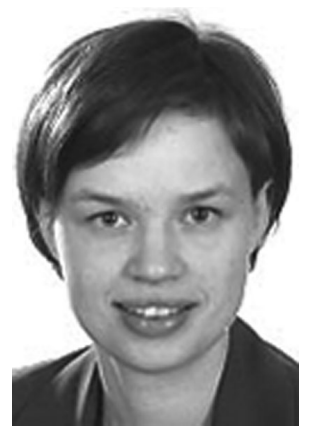

Melanie Luppa

Bibliografie DOI http://dx.doi.org/ 10.1055/s-0033-1343120 Psychiat Prax 2013; 40: 173-175

(c) Georg Thieme Verlag KG Stuttgart · New York ISSN 0303-4259

Korrespondenzadresse Prof. Dr. med. Steffi G. Riedel-Heller Institut für Sozialmedizin, Arbeitsmedizin und Public Health (ISAP)

Philipp-Rosenthal-Straße 55 04103 Leipzig

Steffi.Riedel-Heller@medizin. uni-leipzig.de

\section{Bevölkerungsmedizinische Bedeutung lange unterschätzt \\ $\nabla$}

Depressive Störungen im Alter sind folgenschwer. Sie sind mit Funktionsbeeinträchtigungen, einer reduzierten Lebensqualität, erhöhten Suizidraten sowie einer erhöhten nicht suizidalen Mortalität verbunden.

Obwohl die Erkrankungsgruppe einen festen Platz im Spektrum gerontopsychiatrischer Erkrankungen hat, wurde die bevölkerungsmedizinische Bedeutung für Hochaltrige lange unterschätzt. Die psychiatrisch-epidemiologische Forschung, die sich mit der Häufigkeit sowie der zeitlichen und räumlichen Verteilung psychischer Erkrankungen in der Bevölkerung und deren Risikofaktoren beschäftigt, hat hier viel beizutragen.

\section{Häufigkeit, Verlauf und Riskiofaktoren $\nabla$}

Depressionen gehören nach den Demenzen zu den häufigsten psychischen Störungen im höheren Lebensalter. Schwere Formen, die mit den gängigen Klassifikationssystemen erfasst werden, sind seltener als leichtere. Eine aktuelle Metaanalyse bevölkerungsbasierter Studien ergab eine Prävalenz von 7,2\% (95\%-KI 4,4-10,6\%) für Major Depression bei über 75-Jährigen [1]. Darüber hinaus erlebt eine substanzielle Zahl von Senioren eine depressive Symptomatik, die nicht die geforderten Kriterien des ICD-10 oder DSM-IV erfüllen. Gleichwohl sind die Betroffenen davon beeinträchtigt. Die genannte Metaanalyse ergab eine Prävalenz für depressive Symptomatik, wie sie im Allgemeinen mit Depressionsskalen gemessen wird, von 17,1\% (95\%-KI 9,7-26,1\%) [1]. Eine weitere Metaanalyse, die über 50-Jährige inkludierte kam auf 19,5\% [2]. Die Leipziger Langzeitstudie in der Altenbevölkerung (LEILA75+), eine populationsbasierte Studie, bei der die deutsche Version der Center for Epidemiologic Studies De- pression Scale (CES-D) zum Einsatz kam, ergab eine Prävalenz von 14\% (95\%-KI 12-17\%) [3]. In der AgeCoDe-Studie, der größten laufenden Alterskohortenstudie in Deutschland, waren es, gemessen mit der Geriatric Depression Scale (GDS15), $10 \%$ [4]. Ältere Migranten erscheinen besonders belastet [5]. Eine systematische Übersichtsarbeit fand Neuerkrankungsraten von $0,2-14,1$ pro 100 Personenjahre [6]. Ein substanzieller Teil der Erkrankungen verläuft chronisch. Über einen Beobachtungszeitraum von 8 Jahren zeigten $40 \%$ der Studienteilnehmer einen intermittierenden oder chronischen Verlauf [3]. Eine 2011 publizierte Übersicht unterstreicht die Relevanz von sogenannten unterschwelligen Depressionen, die ein substanzielles Risiko für die Entwicklung einer Major Depression darstellen [7].

Weibliches Geschlecht, funktionelle Beeinträchtigungen aufgrund verschiedener somatischer Erkrankungen, insbesondere Mobilitäts- und Sehbeeinträchtigungen, sowie ein eingeschränktes soziales Netzwerk können als wichtige Risikofaktoren für die Entwicklung von depressiven Symptomen gelten [8-10]. Die Richtung der Assoziation von funktionellen Beeinträchtigungen und depressiven Symptomen war lange unklar. Aktuelle Ergebnisse deuten darauf hin, dass funktionelle Beeinträchtigungen durch somatische Erkrankungen ein Risikofaktor für die Entwicklung depressiver Symptomatik sind und diesen oft vorausgehen.

Zahlreiche Befunde zeigen, dass komorbide depressive Störungen den Verlauf somatischer Erkrankungen, insbesondere kardiovaskulärer Erkrankungen, deutlich negativ beeinflussen [11, 12]. 


\section{Versorgungsepidemiologische Befunde}

$\nabla$

Eine aktuelle Metaanalyse zeigt, dass depressive Störungen im Alter im primärärztlichen Setting unterdiagnostiziert sind. Grob die Hälfte bleibt unerkannt. Dabei wird davon ausgegangen, dass der Grad der Erkennung für alte Menschen deutlich unter dem von Depressionen im jüngeren Alter liegt [13]. Obwohl Depressionen behandelbar sind, werden auch Patienten, die als solche identifiziert werden, nur selten behandelt [14]. Versorgungsepidemiologische Befunde zur psychotherapeutischen Behandlungspraxis für depressive alte Menschen in Deutschland existieren nicht. Das ist wenig verwunderlich.

Trotzdem gehören Menschen mit depressiven Störungen zu den Vielnutzern des Gesundheitssystems, wobei die allerwenigsten eine depressionsspezifische Behandlung erhalten. Eine aktuelle systematische Übersicht zur Inanspruchnahme von Gesundheitsleistungen und Kosten bei Patienten mit depressiven Störungen zeigte durchgehend, dass die ambulanten, stationären sowie die Gesamtkosten depressiver Senioren ein Drittel über den entsprechenden Kosten nicht depressiver Senioren liegen [14]. Erste deutsche Daten zu den direkten Kosten von depressiven Störungen bei über 75-jährigen Allgemeinarztpatienten ergaben durchschnittliche jährliche direkte Gesamtkosten in Höhe von 5241 Euro für Depressive und von 3648 Euro für Nichtdepressive [15]. Obgleich auf unterschiedlichem Kostenniveau angesiedelt, ist dies auch mit den klassischen US-amerikanischen Studien vergleichbar [16-18].

Eine Pilotanalyse zum Verlauf der Versorgungskosten bei depressiver Symptomatik im höheren Alter aus der eigenen Arbeitsgruppe zeigt darüber hinaus, dass leichte depressive Symptome auch die Versorgungskosten in der Zukunft deutlich beeinflussen, unabhängig von vorliegenden körperlichen Erkrankungen [19].

\section{Fazit und Implikationen für die Versorgung $\nabla$}

Aktuelle epidemiologische Forschung unterstreicht die Relevanz depressiver Symptomatik für Hochaltrige. Depressive Störungen im Alter sind behandelbar. Internationale Arbeiten zeigen, dass sowohl psychopharmakologische Interventionen [20, 21], als auch psychotherapeutische Interventionen [22], wie z.B. die speziell für das allgemeinärztliche Setting entwickelte ProblemlöseTherapie (PST-PC) oder die interpersonelle Psychotherapie (IPT), sich für ältere Menschen als praktikabel und effektiv erwiesen haben. In einer deutschen Studie konnte auch für alte Menschen gezeigt werden, dass kognitive Verhaltenstherapie als Gruppentherapie eine gut angenommene, effiziente Behandlungsform darstellt [23]. Andere nicht pharmakologische Interventionen, wie physische Aktivierung und soziale Vernetzung zeigen ermutigende Resultate [24]. In der nationalen Versorgungsleitlinie zur Depression wird die Versorgung depressiver Störungen im Alter nur unzureichend aufgegriffen und nicht systematisch recherchiert [25].

Das Verständnis der Patientenperspektive wird gegenwärtig wenig berücksichtigt. Im Gegensatz zu früheren Annahmen, Senioren würden psychopharmakologische Interventionen präferieren, gibt es international zunehmend Evidenz, dass auch alte Menschen psychosozialen Interventionen einen bevorzugten Stellenwert einräumen [26].

Deutsche Senioren sind mehrheitlich regelmäßig bei ihrem Hausarzt in Behandlung. Er gilt nach wie vor als erster Ansprech- partner für psychische und somatische Probleme älterer Menschen. Eine Metaanalyse zeigt, dass einfache Trainingsprogramme für den allgemeinärztlichen Versorgungsbereich zur Erhöhung der Erkennungs- und Behandlungsraten ineffektiv sind [27] und komplexe Interventionen notwendig werden. International spielen deshalb positiv evaluierte Versorgungsmodelle eine besondere Rolle, die fachspezifische Expertise in das primärärztliche Setting inkludieren [28, 29]. Das IMPACT-Programm ist das am besten untersuchte Programm, das die stärkste Evidenz im Hinblick auf die Wirksamkeit einer kombinierten kollaborativen, gestuften Behandlung zeigte [28]. Niedrigschwellige Interventionen, die Selbstmanagementansätze einschließen und zum Beispiel körperliche Aktivitäten fördern, stellen sinnvolle Weiterentwicklungen dieser Versorgungsmodelle dar [30].

Epidemiologische und versorgungsepidemiologische Forschung zeigt, dass mit der demografischen Entwicklung depressive Störungen im Alter zu einer zentralen und drängenden Versorgungsherausforderung gehören werden. Evidenzbasierte Behandlungsmöglichkeiten und exzellent evaluierte Versorgungsarrangements für alte depressive Menschen liegen international vor. Es besteht dringender Handlungsbedarf, um zur internationalen Entwicklung aufzuschließen.

\section{Danksagung}

Der Beitrag entstand im Zusammenhang mit den Projekten AgeMooDe (Depression im Alter: Versorgungsbedarf, Inanspruchnahme von Gesundheitsleistungen und Kosten, BMBF: 01GY1155A).

\section{Literatur}

1 Luppa M, Sikorski C, LuckT et al. Age- and gender-specific prevalence of depression in latest-life - Systematic review and meta-analysis. J Affect Disord 2012; 136: 212-221

2 Volkert J, Schulz H, Härter $M$ et al. The prevalence of mental disorders in older people in Western countries - a meta-analysis. Ageing Res Rev 2013; $12: 339-353$

3 Luppa M, Luck T, König HH et al. Natural course of depressive symptoms in late life. An 8-year population-based prospective study. J Affect Disord 2012; 142: 166-171

4 Weyerer S, Eifflaender-Gorfer S, Kohler $L$ et al. Prevalence and risk factors for depression in non-demented primary care attenders aged 75 years and older. J Affect Disord 2008; 111: 153-163

5 Aichberger MC, Neuner B, Hapke $U$ et al. Association between migrant status and depressive symptoms in the older population in Germany. Psychiat Prax 2012; 39: 116-121

6 Büchtemann D, Luppa M, Bramesfeld A et al. Incidence of late-life depression: a systematic review. J Affect Disord 2012; 142: 172-179

7 Meeks TW, Vahia IV, Lavretsky H et al. A tune in "a minor" can "b major": A review of epidemiology, illness course, and public health implications of subthreshold depression in older adults. J Affect Disord 2011; 129: 126 - 142

8 Luppa M, Sikorski C, Luck T et al. Prevalence and risk factors of depressive symptoms in latest life - results of the Leipzig Longitudinal Study of the Aged (LEILA 75+). Int J Geriatr Psychiatry 2012; 27: 286-295

9 Weyerer S, Eifflaender-Gorfer S, Wiese B et al. Incidence and predictors of depression in non-demented primary care attenders aged 75 years and older: results from a 3-year follow-up study. Age Ageing 2013; 42: $173-180$

10 Schwarzbach M, Luppa M, Sikorski C et al. The relationship between social integration and depression in non-demented primary care patients aged 75 years and older. J Affect Disord 2013; 145: 172 - 178

11 Blazer DG. Depression in late life: Review and commentary. J Gerontol A Biol Sci Med Sci 2003; 58: 249-265

12 Lehnert T, Konnopka A, Riedel-Heller S et al. Diabetes Mellitus and Comorbid Depression: Economic Findings from a Systematic Literature Review. Psychiat Prax 2011; 38: 369-375

13 Mitchell AJ, Rao S, Vaze A. Do Primary Care Physicians Have Particular Difficulty Identifying Late-Life Depression? A Meta-Analysis Stratified by Age. Psychother Psychosom 2010; 79: 285-294 
14 Luppa M, Sikorski C, Motzek T et al. Health Service Utilization and Costs of Depressive Symptoms in Late Life - A Systematic Review. Curr Pharm Des 2012; 18: 5936-5957

15 Luppa M, Heinrich S, Matschinger $H$ et al. Direct costs associated with depression in old age in Germany. J Affect Disord 2008; 105: 195 - 204

16 Katon WJ, Lin E, Russo J et al. Increased medical costs of a populationbased sample of depressed elderly patients. Arch Gen Psychiatry 2003; 60: 897-903

17 Luber MP, Meyers BS, Williams-Russo PG et al. Depression and service utilization in elderly primary care patients. Am J Geriatr Psychiatry 2001; 9: 169-176

18 Unützer J, Patrick DL, Simon G et al. Depressive symptoms and the cost of health services in HMO patients aged 65 years and older - A 4-year prospective study. JAMA 1997; 277: 1618 - 1623

19 Luppa M, König HH, Heider D et al. Direct costs associated with depressive symptoms in late life - a 4.5 year prospective study. Int Psychogeriatr 2013; 25: $292-302$

20 Wilson K, Mottram P, Sivanranthan A et al. Antidepressant versus placebo for depressed elderly. Cochrane Database Syst Rev 2001; 2: CD000561

21 Gill D, Hatcher S. Antidepressants for depression in medical illness. Cochrane Database Syst Rev 2000; 4: CD001312
22 Wolf NJ, Hopko DR. Psychosocial and pharmacological interventions for depressed adults in primary care: A critical review. Clin Psychol Rev 2008; 28: 131 - 161

23 Hautzinger M, Welz S. Kognitive Verhaltenstherapie bei Depression im Alter. Z Gerontol Geriat 2004; 37: 427-435

24 Blake H, Mo P, Malik S et al. How effective are physical activity interventions for alleviating depressive symptoms in older people? A systematic review. Clin Rehab 2009; 23: 873-887

25 DGPPN, BÄK, KBV, AWMF Hrsg. Nationale Versorgungsleitlinie Unipolare Depression. Reihe: Interdisziplinäre S3-Praxisleitlinien. Berlin: Springer; 2010

26 Gum AM, Arean PA, Hunkeler E et al. Depression treatment preferences in older primary care patients. Gerontologist 2006; 46: 14-22

27 Sikorski C, Luppa M, König HH et al. Does GP training in depression care affect patient outcome? - A systematic review and meta-analysis. BMC Health Serv Res 2012; 12: 10

28 Skultety KM, Zeiss A. The treatment of depression in older adults in the primary care setting: An evidence-based review. Health Psychol 2006; 25: $665-674$

29 Skultety KM, Rodriguez RL. Treating geriatric depression in primary care. Curr Psychiatry Rep 2008; 10: 44-50

30 Yeung A, Geldman G, Fava M. Self-Management of Depression. Cambridge: Cambridge University Press; 2010 